\title{
An Improved Nonequidistant Grey Model Based on Simpson Formula and Its Application
}

\author{
Zhiming $\mathrm{Hu}$ (D) $^{1}$ and Chong Liu $\mathbb{D}^{2}$ \\ ${ }^{1}$ School of Zhejiang College, Shanghai University of Finance \& Economics, Jinhua 321013, China \\ ${ }^{2}$ School of Science, Inner Mongolia Agricultural University, Hohhot 010018, China \\ Correspondence should be addressed to Chong Liu; liuchong@emails.imau.edu.cn
}

Received 1 November 2020; Revised 13 April 2021; Accepted 21 April 2021; Published 29 April 2021

Academic Editor: Pietro De Lellis

Copyright $\odot 2021$ Zhiming Hu and Chong Liu. This is an open access article distributed under the Creative Commons Attribution License, which permits unrestricted use, distribution, and reproduction in any medium, provided the original work is properly cited.

\begin{abstract}
Grey prediction models have been widely used in various fields of society due to their high prediction accuracy; accordingly, there exists a vast majority of grey models for equidistant sequences; however, limited research is focusing on nonequidistant sequence. The development of nonequidistant grey prediction models is very slow due to their complex modeling mechanism. In order to further expand the grey system theory, a new nonequidistant grey prediction model is established in this paper. To further improve the prediction accuracy of the NEGM $\left(1,1, t^{2}\right)$ model, the background values of the improved nonequidistant grey model are optimized based on Simpson formula, which is abbreviated as INEGM $\left(1,1, t^{2}\right)$. Meanwhile, to verify the validity of the proposed model, this model is applied in two real-world cases in comparison with three other benchmark models, and the modeling results are evaluated through several commonly used indicators. The results of two cases show that the INEGM $\left(1,1, t^{2}\right)$ model has the best prediction performance among these competitive models.
\end{abstract}

\section{Introduction}

Time-series forecasting has received extensive attention in the past decades. Accordingly, there exist a plenty of approaches for time-series analysis and forecasting. From $[1,2]$ and the references therein, these methods can be divided into three categories: statistical methods (e.g., regression analysis [3], functional state space model [4], logistic regression [5], spatial-temporal model [6], Markov chain model [7], etc.), machine learning methods [8-11], and grey modeling technique [12-14]. Each method has its own advantages and limitations [15]. Regarding statistical methods and machine learning methods, the growing body of data is required in the modeling procedure. However, it is difficult to collect data available for model calibration in practical applications [16-18]. This is the principal reason that the grey model has been widely used in various disciplines $[19,20]$.

Grey prediction models proposed by Deng [21] have been widely used in various fields of society due to their high prediction accuracy. In particular, the GM $\left(1,1, t^{2}\right)$ model is an important branch of the grey prediction model, which was pioneered by Qian et al. [22]. To further improve the prediction accuracy and applicability of the GM $\left(1,1, t^{2}\right)$ model, many scholars have made efforts. For example, Luo and Wei [23] constructed the expectation function by using the sum of error squares and solved the expression of the optimal constant in the time response function. Considering that the background values are key factor that affects the prediction accuracy of the GM $\left(1,1, t^{2}\right)$ model, Wei et al. [24] used the linear interpolation method to reconstruct the background values of the GM $\left(1,1, t^{2}\right)$ model. Although these studies have improved the prediction accuracy of the GM $\left(1,1, t^{2}\right)$ model in the field of equidistant time series, to the best of our knowledge, there is a dearth of research on nonequidistant time series. However, in our real world, there exist a large number of nonequidistant time series, such as samplings in reaction furnaces and building settlements. On this basis, this paper develops a novel nonequidistant grey model by combining the existing GM $\left(1,1, t^{2}\right)$ model and 
concept of nonequidistant time series. To further enhance the prediction performance of the nonequidistant GM $(1,1$, $t^{2}$ ) model, a Simpson formula is applied to optimize the background value of the nonequidistant GM $\left(1,1, t^{2}\right)$ model; as a result, an improved nonequidistant GM $\left(1,1, t^{2}\right)$ model (denoted as INEGM $\left(1,1, t^{2}\right)$ ) is proposed in this paper. The main contributions of this paper are summarized as follows:

(1) A conventional nonequidistant GM $\left(1,1, t^{2}\right)$ model is constructed by incorporating the concept of nonequidistant time series into the GM $\left(1,1, t^{2}\right)$ model already in place

(2) Simpson formula is applied to optimize the background value of the conventional nonequidistant GM $\left(1,1, t^{2}\right)$ model to increase the prediction performance

(3) Two real-world cases are used to verify the validity and superiority of the proposed model in comparison with other benchmark models

The rest of this paper is organized as follows. Section 2 introduces the conventional nonequidistant GM $\left(1,1, t^{2}\right)$ model and analyzes the discretization error. Section 3 optimizes the nonequidistant GM $\left(1,1, t^{2}\right)$ model by using a Simpson formula. Section 4 verifies the applicability of the proposed model and Section 5 concludes the paper.

\section{Classic Nonequidistant $G M\left(1,1, t^{2}\right)$ Model and Its Error Analysis}

2.1. Nonequidistant $G M\left(1,1, t^{2}\right)$ Model. In accordance with the description in [25], it is easy to establish the nonequidistant GM $\left(1,1, t^{2}\right)$ model (denoted as NEGM $\left(1,1, t^{2}\right)$ ), whose modeling process can be outlined as follows.

Step 1. Suppose that the original time series is $X^{(0)}=\left(x^{(0)}\left(k_{1}\right), x^{(0)}\left(k_{2}\right), \ldots, x^{(0)}\left(k_{n}\right)\right)$, where $\Delta k_{i}$ $=k_{i}-k_{i-1} \neq$ const, $i=2,3, \ldots, n$; $\quad$ then $\quad X^{(1)}=\left(x^{(1)}\right.$ $\left.\left(k_{1}\right), x^{(1)}\left(k_{2}\right), \ldots, x^{(1)}\left(k_{n}\right)\right)$ denotes the first-order accumulated generating operation sequence of $X^{(0)}$, where

$$
x^{(1)}\left(k_{i}\right)=\sum_{j=1}^{i} x^{(0)}\left(k_{j}\right), \quad i=1,2, \ldots, n .
$$

Step 2. The differential equation of NEGM $\left(1,1, t^{2}\right)$ model is expressed as

$$
\frac{\mathrm{d} x^{(1)}(t)}{\mathrm{d} t}+a x^{(1)}(t)=b t^{2}+c t+d .
$$

Then, we get the discrete formula of equation (2) expressed as

$$
x^{(1)}\left(k_{i}\right)-x^{(1)}\left(k_{i-1}\right)+a z^{(1)}\left(k_{i}\right)=\frac{\left(k_{i}^{3}-k_{i-1}^{3}\right) b}{3}+\frac{\left(k_{i}^{2}-k_{i-1}^{2}\right) c}{2}+\mathrm{d} \Delta k_{i},
$$

where $z^{(1)}\left(k_{i}\right)$ is the background value and $z^{(1)}\left(k_{i}\right)=0.5\left(x^{(1)}\left(k_{i}\right)+x^{(1)}\left(k_{i-1}\right)\right), k=2,3, \ldots, n$.

Step 3. The model parameters can be calculated as

$$
(a, b, c, d)^{T}=\left(B^{T} B\right)^{-1} B^{T} Y,
$$

where

$$
\begin{gathered}
B=\left[\begin{array}{cccc}
-z^{(1)}\left(k_{2}\right) \Delta k_{2} & \frac{1}{3}\left(k_{2}^{3}-k_{1}^{3}\right) & \frac{1}{2}\left(k_{2}^{2}-k_{1}^{2}\right) & \Delta k_{2} \\
-z^{(1)}\left(k_{3}\right) \Delta k_{3} & \frac{1}{3}\left(k_{3}^{3}-k_{2}^{3}\right) & \frac{1}{2}\left(k_{3}^{2}-k_{2}^{2}\right) & \Delta k_{3} \\
\vdots & \vdots & \vdots & \vdots \\
-z^{(1)}\left(k_{n}\right) \Delta k_{n} & \frac{1}{3}\left(k_{n}^{3}-k_{n-1}^{3}\right) & \frac{1}{2}\left(k_{n}^{2}-k_{n-1}^{2}\right) & \Delta k_{n}
\end{array}\right], \\
Y=\left[x^{(0)}\left(k_{2}\right) \Delta k_{2}, x^{(0)}\left(k_{3}\right) \Delta k_{3}, \ldots, x^{(0)}\left(k_{n}\right) \Delta k_{n}\right]^{T} .
\end{gathered}
$$

Step 4. The time response function of equation (2) is expressed as

$$
\begin{aligned}
\tilde{x}^{(1)}\left(k_{i}\right)= & e^{-a\left(k_{i}-k_{1}\right)}\left(x^{(1)}\left(k_{1}\right)-\left(\frac{b}{a} k_{1}^{2}+\frac{a c-2 b}{a^{2}} k_{1}\right.\right. \\
& \left.\left.+\frac{2 b-a c+a^{2} d}{a^{3}}\right)\right) \\
= & \frac{b}{a} k_{i}^{2}+\frac{a c-2 b}{a^{2}} k_{i}+\frac{2 b-a c+a^{2} d}{a^{3}} .
\end{aligned}
$$

Step 5. The predicted values of the original sequence are expressed as

$$
\tilde{x}^{(0)}\left(k_{i}\right)= \begin{cases}\frac{\tilde{x}^{(1)}\left(k_{i}\right)-\tilde{x}^{(1)}\left(k_{i-1}\right)}{\Delta k_{i}}, & i=2,3, \ldots, \\ x^{(0)}\left(k_{i}\right), & i=1 .\end{cases}
$$


2.2. Error Analysis. Having reviewed the above modeling procedure, it is noticed that the prediction accuracy of NEGM $\left(1,1, t^{2}\right)$ model depends on the model parameters that are closely related to the background value; thus the background value is essential for the prediction precision. In the classic NEGM $\left(1,1, t^{2}\right)$ model, both sides of equation (2) are integrated over the interval $\left[k_{i-1}, k_{i}\right]$, expressed as

$$
\begin{gathered}
\int \frac{\mathrm{d} x^{(1)}}{\mathrm{d} t} \mathrm{~d} t+a \int x^{(1)} \mathrm{d} t=\int\left(b t^{2}+c t+d\right) \mathrm{d} t \Rightarrow \\
x^{(1)}\left(k_{i}\right)-x^{(1)}\left(k_{i-1}\right)+a \int x^{(1)} \mathrm{d} t=\frac{\left(k_{i}^{3}-k_{i-1}^{3}\right) b}{3}+\frac{\left(k_{i}^{2}-k_{i-1}^{2}\right) c}{2}+\mathrm{d} \Delta k_{i} .
\end{gathered}
$$

We observe from equations (3) and (9) that the discretization method, so-called trapezoid formula, is employed to approximately calculate the integral $\int x^{(1)} \mathrm{d} t$, thus producing the discretization error in such a transition process. To be specific, when the above integral has a concave trend, the approximate value is larger than the actual one; when the above integral has an upward convex trend, the approximate value is lower than the actual one.

\section{Presentation of INEGM $\left(1,1, t^{2}\right)$}

3.1. Nonequidistant Simpson Numerical Integral. To decline the discretization error mentioned in Section 2.2, this section applies the concept of function approximation to calculate the area of the curved trapezoid. Since the integrable function is easily approached by the Lagrange function, we get a Lagrange polynomial $\psi(x)$ over the interval $a \leq x_{1}<x_{2}<\cdots \leq b$ denoted as $L_{n}(x)$, where

$$
L_{n}(x)=\sum_{j=1}^{n+1} l_{j}(x) f\left(x_{j}\right)
$$

where $l_{j}(x)$ is the fundamental polynomial.

Definition 1 (see [26]). If the n-order polynomial

$$
l_{j}(x), \quad j=0,1, \ldots, n,
$$

satisfies

$$
l_{j}(x)=\left\{\begin{array}{ll}
1, & k=j, \\
0, & k \neq j,
\end{array}, \quad j, k=0,1, \ldots, n .\right.
$$

on nodes $\quad x_{0}<x_{1}<x_{2}<\cdots<x_{n}$, then $l_{0}(x), l_{1}(x), \ldots, l_{n}(x)$, are all interpolation basis functions on nodes $x_{0}, x_{1}, x_{2}, \ldots, x_{n}$. Lagrange interpolation polynomial is expressed as

$$
L_{n}(x)=\sum_{k=0}^{n} f\left(x_{k}\right) \frac{w_{n+1}(x)}{\left(x-x_{k}\right) w_{n+1}^{\prime}\left(x_{k}\right)},
$$

where

$$
\begin{aligned}
w_{n+1}(x) & =\left(x-x_{0}\right)\left(x-x_{1}\right) \cdots\left(x-x_{n}\right), \\
w_{n+1}^{\prime}\left(x_{k}\right) & =\left(x_{k}-x_{0}\right)\left(x_{k}-x_{1}\right) \cdots\left(x_{k}-x_{n}\right) .
\end{aligned}
$$

Therefore,

$$
f(x) \approx \sum_{j=1}^{n+1} l_{j}(x) f\left(x_{j}\right)
$$

By integrating both sides of equation (14), we obtain

$$
I_{n}[f]=\sum_{j=1}^{n+1} A_{j} f\left(x_{j}\right),
$$

where $A_{j}=\int l_{j}(x) \mathrm{d} x$ is the interpolation coefficient and $I_{n}[f]$ denotes the interpolation quadrature formula.

Theorem 1. Assume that

$$
\int_{k_{i-1}}^{k_{i+1}} x^{(1)}(t) \mathrm{d} t=I_{i-1, i+1}\left[x^{(1)}\right]
$$

and then the nonequidistant Simpson numerical integral formula is given as

$$
I_{i-1, i+1}\left[x^{(1)}\right] \approx \frac{\Delta k_{i}+\Delta k_{i+1}}{6}\left(\frac{2 \Delta k_{i}-\Delta k_{i+1}}{\Delta k_{i}} x_{i-1}^{(1)}+\frac{\left(\Delta k_{i}+\Delta k_{i+1}\right)^{2}}{\Delta k_{i} \Delta k_{i+1}} x_{i}^{(1)}+\frac{2 \Delta k_{i+1}-\Delta k_{i}}{\Delta k_{i+1}} x_{i+1}^{(1)}\right) .
$$

Proof. By integrating $x^{(1)}(t)$ over the interval $\left[k_{i-1}, k_{i+1}\right], i=2,3, \ldots, n-1$, we have

$$
I_{i-1, i+1}\left[x^{(1)}\right] \approx \sum_{m=i-1}^{i+1} A_{m} x^{(1)}\left(k_{m}\right)=\sum_{m=i-1}^{i+1} A_{m} x_{m}^{(1)} .
$$

$$
\begin{aligned}
l_{m}(k) & =\prod_{\substack{j=1 \\
j \neq m}}^{i+1} \frac{k-k_{j}}{k_{m}-k_{j}}, \\
A_{m} & =\int_{k-1}^{k+1} l_{m}(x) \mathrm{d} x .
\end{aligned}
$$


When $m=i-1$, we have

$$
A_{i-1}=\int_{k_{i-1}}^{k_{i+1}} l_{i-1}(k) \mathrm{d} k=\int_{k_{i-1}}^{k_{i+1}} \frac{\left(k-k_{i}\right)\left(k-k_{i+1}\right)}{\left(k_{i-1}-k_{i}\right)\left(k_{i-1}-k_{i+1}\right)} \mathrm{d} k=\frac{\left(\Delta k_{i}+\Delta k_{i+1}\right)\left(2 \Delta k_{i}-\Delta k_{i+1}\right)}{6 \Delta k_{i}} .
$$

When $m=i, m=i+1$, we have

Thus,

$$
\begin{aligned}
A_{i} & =\frac{\left(\Delta k_{i}+\Delta k_{i+1}\right)^{3}}{6 \Delta k_{i} \Delta k_{i+1}}, \\
A_{i+1} & =\frac{\left(\Delta k_{i}+\Delta k_{i+1}\right)\left(2 \Delta k_{i+1}-\Delta k_{i}\right)}{6 \Delta k_{i+1}} .
\end{aligned}
$$

$$
I_{i-1, i+1}\left[x^{(1)}\right] \approx \frac{\Delta k_{i}+\Delta k_{i+1}}{6}\left(\frac{2 \Delta k_{i}-\Delta k_{i+1}}{\Delta k_{i}} x_{i-1}^{(1)}+\frac{\left(\Delta k_{i}+\Delta k_{i+1}\right)^{2}}{\Delta k_{i} \Delta k_{i+1}} x_{i}^{(1)}+\frac{2 \Delta k_{i+1}-\Delta k_{i}}{\Delta k_{i+1}} x_{i+1}^{(1)}\right) .
$$

It is proved.

$$
\int \mathrm{d} x^{(1)}+a \int x^{(1)}(t) \mathrm{d} t=\int\left(b t^{2}+c t+d\right) \mathrm{d} t .
$$

3.2. Model Establishment. By integrating both sides of According to equation (1), we have equation (2) over the interval $\left[k_{i-1}, k_{i+1}\right]$, we get

$$
\int_{k_{i-1}}^{k_{i+1}} \mathrm{~d} x^{(1)}(t)=x^{(1)}\left(k_{i+1}\right)-x^{(1)}\left(k_{i-1}\right)=x^{(0)}\left(k_{i+1}\right) \Delta k_{i+1}+x^{(0)}\left(k_{i}\right) \Delta k_{i}
$$

Furthermore,

$$
x^{(0)}\left(k_{i+1}\right) \Delta k_{i+1}+x^{(0)}\left(k_{i}\right) \Delta k_{i}+a \int_{k_{i-1}}^{k_{i+1}} x^{(1)}(t) \mathrm{d} t=\frac{b}{3}\left(k_{i+1}^{3}-k_{i-1}^{3}\right)+\frac{c}{2}\left(k_{i+1}^{2}-k_{i-1}^{2}\right)+\mathrm{d}\left(\Delta k_{i+1}+\Delta k_{i}\right) .
$$

Then, we replace $\int_{k_{i-1}}^{k_{i+1}} x^{(1)}(t) \mathrm{d} t$ with the nonequidistant Simpson numerical integral formula, which yields that

$$
\begin{aligned}
& x^{(0)}\left(k_{i+1}\right) \Delta k_{i+1}+x^{(0)}\left(k_{i}\right) \Delta k_{i}+a \frac{\Delta k_{i}+\Delta k_{i+1}}{6}\left(\frac{2 \Delta k_{i}-\Delta k_{i+1}}{\Delta k_{i}} x_{i-1}^{(1)}+\frac{\left(\Delta k_{i}+\Delta k_{i+1}\right)^{2}}{\Delta k_{i} \Delta k_{i+1}} x_{i}^{(1)}+\frac{2 \Delta k_{i+1}-\Delta k_{i}}{\Delta k_{i+1}} x_{i+1}^{(1)}\right) \\
& =\frac{b}{3}\left(k_{i+1}^{3}-k_{i-1}^{3}\right)+\frac{c}{2}\left(k_{i+1}^{2}-k_{i-1}^{2}\right)+\mathrm{d}\left(\Delta k_{i+1}+\Delta k_{i}\right) .
\end{aligned}
$$

Then, the model parameters can be obtained by the leastsquare method expressed as

$$
(a, b, c, d)^{T}=\left(\vartheta^{T} \vartheta\right)^{-1} \vartheta^{T} \omega,
$$

where 


$$
\begin{aligned}
& \vartheta=\left[\begin{array}{cccc}
-I_{1,3}\left[x^{(1)}\right] & \frac{1}{3}\left(k_{2}^{3}-k_{1}^{3}\right) & \frac{1}{2}\left(k_{2}^{2}-k_{1}^{2}\right) & \Delta k_{2}+\Delta k_{3} \\
-I_{2,4}\left[x^{(1)}\right] & \frac{1}{3}\left(k_{3}^{3}-k_{2}^{3}\right) & \frac{1}{2}\left(k_{3}^{2}-k_{2}^{2}\right) & \Delta k_{3}+\Delta k_{4} \\
\vdots & \vdots & \vdots & \vdots \\
-I_{n-2, n}\left[x^{(1)}\right] & \frac{1}{3}\left(k_{n}^{3}-k_{n-1}^{3}\right) & \frac{1}{2}\left(k_{n}^{2}-k_{n-1}^{2}\right) & \Delta k_{n-1}+\Delta k_{n}
\end{array}\right] \\
& \omega=\left[\begin{array}{c}
x^{(0)}\left(k_{2}\right) \Delta k_{2}+x^{(0)}\left(k_{3}\right) \Delta k_{3} \\
x^{(0)}\left(k_{3}\right) \Delta k_{3}+x^{(0)}\left(k_{4}\right) \Delta k_{4} \\
\vdots \\
x^{(0)}\left(k_{n-1}\right) \Delta k_{n}+x^{(0)}\left(k_{n}\right) \Delta k_{n}
\end{array}\right]
\end{aligned}
$$

It is noticed that the methods for time response function and predicted values of the improved model are similar to the classic nonequidistant nonhomogeneous grey model (elaborated on in Section 2).

3.3. Evaluation Model Indicator. In this section, seven statistical metrics are introduced to examine the prediction performance of the proposed model, as shown in Table 1 .

\section{Numerical Experiment}

In this section, two real-world examples are used to demonstrate the superiority of the proposed model in comparison with other benchmark models including the GM (1, $1)$, NGM $(1,1, k)$, and NEGM $\left(1,1, t^{2}\right)$ models.

Case 1. In this case, monitoring data of building's settlement point, seen in Table 2, are considered as an example to examine the prediction accuracy of the proposed model. It is noticed that the first seven pieces of data are used for calibrating these competitive models and the remaining six pieces of data are used to validate the prediction performance.

In accordance with the current study and references herein, the time response functions of the competitive models are calculated as follows:

(1) GM $(1,1)$

$$
\hat{x}^{(1)}\left(k_{i}\right)=23160.665572 e^{0.003405\left(k_{i}-k_{1}\right)}-23082.05572 .
$$

(2) $\operatorname{NGM}(1,1, k)$

$$
\widehat{x}^{(1)}\left(k_{i}\right)=22.79845 e^{-0.19278\left(k_{i}-k_{1}\right)}-82.31431 k_{i}-26.51276 .
$$

(3) $\operatorname{NEGM}\left(1,1, t^{2}\right)$

$$
\begin{aligned}
\widehat{x}^{(1)}\left(k_{i}\right)= & 2.52708 e^{-1.15026\left(k_{i}-k_{1}\right)}-0.09669 k_{i}^{2} \\
& -79.42318 k_{i}-3.44695 .
\end{aligned}
$$

(4) $\operatorname{INEGM}\left(1,1, t^{2}\right)$

$$
\begin{aligned}
\widehat{x}^{(1)}\left(k_{i}\right)= & 2.63554 e^{-2.22606\left(k_{i}-k_{1}\right)}-0.09426 k_{i}^{2} \\
& -79.44938 k_{i}-3.57919 .
\end{aligned}
$$

We observe from Tables 3 and 4 and Figure 1 that the predicted values of the NGM $(1,1, k)$ model are lower than the actual one, while the predicted values of the GM $(1,1)$ model are higher than the actual one. Meanwhile, the six indicators of the proposed model are better than those of other benchmarks as a whole; therefore, the proposed model has a better prediction performance than those of other competitive models in this experiment.

Case 2. This case takes an example collected from the literature [28] to illustrate the applicability of the proposed model, as shown in Table 5. Similar to Case 1, modeling data is divided into two groups: the first six data sets are applied for model calibration and the remaining four data sets are used to demonstrate the prediction performance of the proposed model.

The time response functions of these competitors are calculated as follows:

(1) GM $(1,1)$

$$
\widehat{x}^{(1)}\left(k_{i}\right)=10116.41987 e^{0.001062\left(k_{i}-k_{1}\right)}-10107.13987 .
$$

(2) $\operatorname{NGM}(1,1, k)$

$$
\begin{aligned}
\widehat{x}^{(1)}\left(k_{i}\right)= & 186.20269 e^{-0.012001\left(k_{i}-k_{1}\right)}-12.68897 k_{i} \\
& -189.61166 .
\end{aligned}
$$

(3) $\operatorname{NEGM}\left(1,1, t^{2}\right)$

$$
\begin{aligned}
\hat{x}^{(1)}\left(k_{i}\right)= & 25.12103 e^{-0.03456\left(k_{i}-k_{1}\right)}+0.003836 k_{i}^{2} \\
& +11.23871 k_{i}-27.08358 .
\end{aligned}
$$

(4) $\operatorname{INEGM}\left(1,1, t^{2}\right)$

$$
\begin{aligned}
\widehat{x}^{(1)}\left(k_{i}\right)= & 14.719505 e^{-0.05473\left(k_{i}-k_{1}\right)}-0.0046978 k_{i}^{2} \\
& -11.04246 k_{i}-16.48645 .
\end{aligned}
$$

After a simple calculation, the predicted values of the original sequence by the four competitive models are tabulated in Table 6, and the relevant error-value metrics are listed in Table 7; for the intuition purpose, these values in Table 7 are plotted in Figure 2. 
TABLE 1: Error-value metrics of the prediction model.

\begin{tabular}{|c|c|}
\hline Index & Formula \\
\hline $\begin{array}{l}\text { Absolute percentage error } \\
\text { Mean absolute percentage error } \\
\text { Mean absolute error }\end{array}$ & $\begin{array}{c}\mathrm{APE}=\left(\left|\widehat{x}^{(0)}(k)-x^{(0)}(k)\right| / x^{(0)}(k)\right) \times 100 \% \\
\mathrm{MAPE}=(1 / n) \sum_{k=1}^{n}\left(\left|\widehat{x}^{(0)}(k)-x^{(0)}(k)\right| / x^{(0)}(k)\right) \times 100 \% \\
\operatorname{MAE}=(1 / n) \sum_{k=1}^{n}\left|\widehat{x}^{(0)}(k)-x^{(0)}(k)\right|\end{array}$ \\
\hline Mean square error & $\operatorname{MAE}=(1 / n) \sum_{k=1}^{n}\left(\widehat{x}^{(0)}(k)-x^{(0)}(k)\right)^{2}$ \\
\hline Root mean square percentage error & $\operatorname{RMSPE}=\sqrt{(1 / n) \sum_{k=1}^{n}\left(\left(\hat{x}^{(0)}(k)-x^{(0)}(k)\right) / x^{(0)}(k)\right)^{2}} \times 100 \%$ \\
\hline Index of agreement & $\mathrm{IA}=1-\left(\sum_{k=1}^{n}\left(\hat{x}^{(0)}(k)-x^{(0)}(k)\right)^{2} / \sum_{k=1}^{n}\left(\left|\bar{x}-x^{(0)}(k)\right|+\left|\hat{x}^{(0)}(k)-\bar{x}\right|\right)^{2}\right)$ \\
\hline Correlation coefficient & $R=\operatorname{Cov}\left(\hat{x}^{(0)}(k), x^{(0)}(k)\right) /\left(\sqrt{\operatorname{Var}\left(\hat{x}^{(0)}(k)\right)} \sqrt{\operatorname{Var}\left(x^{(0)}(k)\right)}\right)$ \\
\hline
\end{tabular}

TABLE 2: Monitoring data of building's settlement point [27].

\begin{tabular}{ll}
\hline Time & Data \\
\hline 1 & 78.60 \\
3 & 78.40 \\
5 & 80.30 \\
8 & 80.70 \\
10 & 81.10 \\
12 & 81.50 \\
15 & 82.10 \\
17 & 82.60 \\
19 & 82.80 \\
22 & 83.70 \\
25 & 83.80 \\
29 & 84.00 \\
31 & 84.70 \\
\hline
\end{tabular}

TABLE 3: Simulative and predictive values by the competitive models in Case 1.

\begin{tabular}{|c|c|c|c|c|c|c|c|c|c|}
\hline \multirow{2}{*}{ Time } & \multirow{2}{*}{ Data } & \multicolumn{2}{|c|}{ GM $(1,1)$} & \multicolumn{2}{|c|}{$\operatorname{NGM}(1,1, k)$} & \multicolumn{2}{|c|}{$\operatorname{NEGM}\left(1,1, t^{2}\right)$} & \multicolumn{2}{|c|}{$\operatorname{INEGM}\left(1,1, t^{2}\right)$} \\
\hline & & Value & APE (\%) & Value & APE (\%) & Value & APE (\%) & Value & APE (\%) \\
\hline \multicolumn{10}{|c|}{ In-sample } \\
\hline 1 & 78.60 & 78.60 & 0.00 & 78.60 & 0.00 & 78.60 & 0.00 & 78.60 & 0.00 \\
\hline 3 & 78.40 & 79.06 & 0.84 & 78.67 & 0.34 & 78.67 & 0.35 & 78.52 & 0.16 \\
\hline 5 & 80.30 & 79.65 & 0.81 & 79.83 & 0.58 & 80.08 & 0.27 & 80.19 & 0.14 \\
\hline 8 & 80.70 & 80.39 & 0.38 & 80.77 & 0.09 & 80.67 & 0.04 & 80.67 & 0.03 \\
\hline 10 & 81.10 & 81.15 & 0.06 & 81.37 & 0.33 & 81.66 & 0.08 & 81.15 & 0.03 \\
\hline 12 & 81.50 & 81.75 & 0.31 & 81.67 & 0.21 & 81.55 & 0.06 & 81.52 & 0.03 \\
\hline 15 & 82.10 & 82.03 & 0.08 & 81.91 & 0.23 & 82.03 & 0.08 & 81.99 & 0.13 \\
\hline \multicolumn{10}{|c|}{ Out-of-sample } \\
\hline 17 & 82.60 & 82.51 & 0.10 & 82.07 & 0.64 & 82.52 & 0.10 & 82.47 & 0.16 \\
\hline 19 & 82.80 & 83.29 & 0.59 & 82.15 & 0.79 & 82.90 & 0.13 & 82.84 & 0.05 \\
\hline 22 & 83.70 & 83.92 & 0.26 & 28.21 & 1.78 & 83.39 & 0.37 & 83.31 & 0.46 \\
\hline 25 & 83.80 & 84.70 & 1.08 & 82.26 & 1.84 & 83.97 & 0.20 & 83.88 & 0.10 \\
\hline 29 & 84.00 & 85.65 & 1.97 & 82.28 & 2.04 & 84.64 & 0.77 & 84.54 & 0.64 \\
\hline 31 & 84.70 & 86.78 & 2.46 & 82.30 & 2.84 & 85.22 & 0.62 & 85.11 & 0.48 \\
\hline
\end{tabular}

We know from Table 7 and Figure 2 that, in the simulation period, the indicators of the proposed model, except the correlation coefficient, are better than those of other benchmarks including the $\operatorname{GM}(1,1), \operatorname{NGM}(1,1, k)$, and NEGM $\left(1,1, t^{2}\right)$ models. Meanwhile, in the prediction period, the indicators of the proposed model, namely MAPE, RMSPE, MAE, MSE, and IA, are $1.6697 \%$, $0.0440 \%, 0.2208,0.0758$, and 0.9065 , respectively, which 
TABle 4: Errors by the competitive models in Case 1.

\begin{tabular}{|c|c|c|c|c|}
\hline Index & GM $(1,1)$ & $\operatorname{NGM}(1,1, k)$ & $\operatorname{NEGM}\left(1,1, t^{2}\right)$ & INEGM $\left(1,1, t^{2}\right)$ \\
\hline \multicolumn{5}{|l|}{ In-sample } \\
\hline MAPE (\%) & 0.4129 & 0.2960 & 0.1456 & 0.0904 \\
\hline RMSPE (\%) & 0.0027 & 0.0011 & 0.0035 & 0.0001 \\
\hline$M A E$ & 0.3301 & 0.2382 & 0.1163 & 0.0726 \\
\hline$M S E$ & 0.1701 & 0.0716 & 0.0222 & 0.0070 \\
\hline$I A$ & 0.9675 & 0.9873 & 0.9960 & 0.9987 \\
\hline$R$ & 0.9564 & 0.9819 & 0.9951 & 0.9986 \\
\hline \multicolumn{5}{|c|}{ Out-of-sample } \\
\hline MAPE (\%) & 1.0750 & 1.6552 & 0.3643 & 0.3152 \\
\hline RMSPE (\%) & 0.0192 & 0.0330 & 0.0020 & 0.0015 \\
\hline$M A E$ & 0.9039 & 1.3891 & 0.3060 & 0.2646 \\
\hline$M S E$ & 1.3616 & 2.3374 & 0.1390 & 0.1051 \\
\hline$I A$ & 0.7427 & 0.4344 & 0.9495 & 0.9604 \\
\hline$R$ & 0.9618 & 0.9437 & 0.9599 & 0.9599 \\
\hline
\end{tabular}

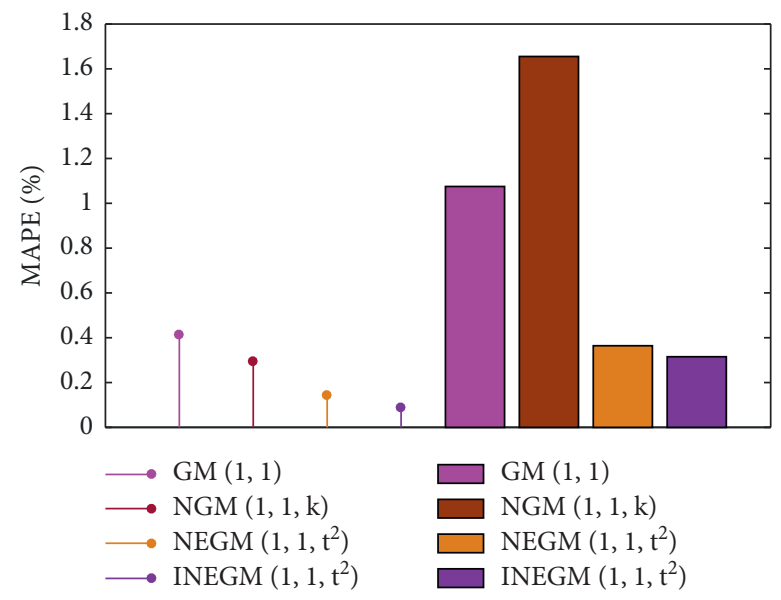

(a)

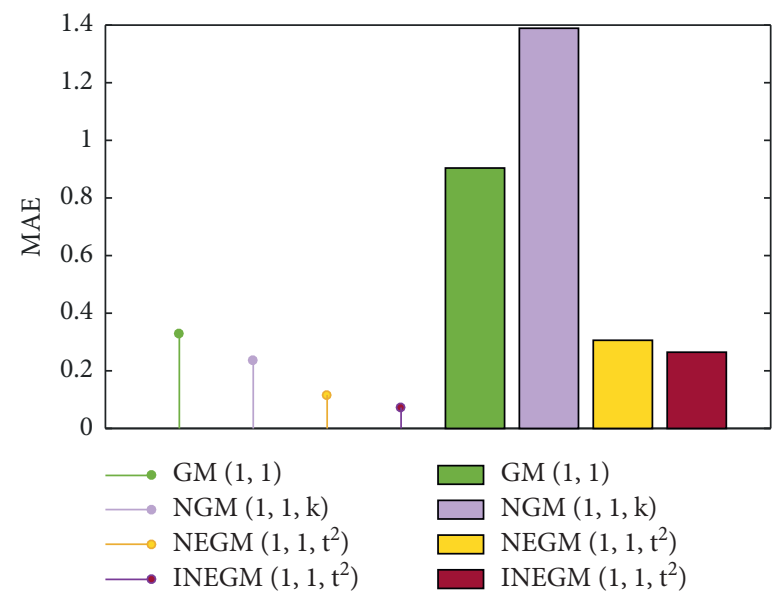

(c)

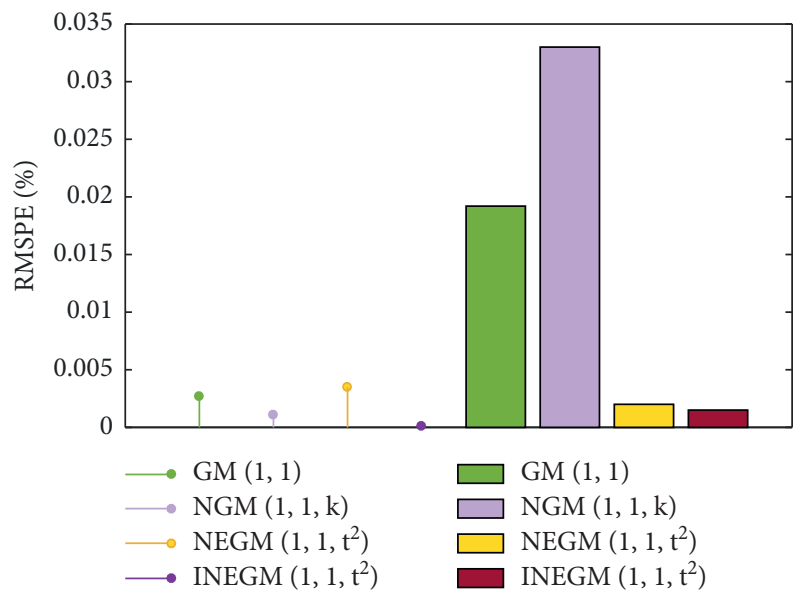

(b)

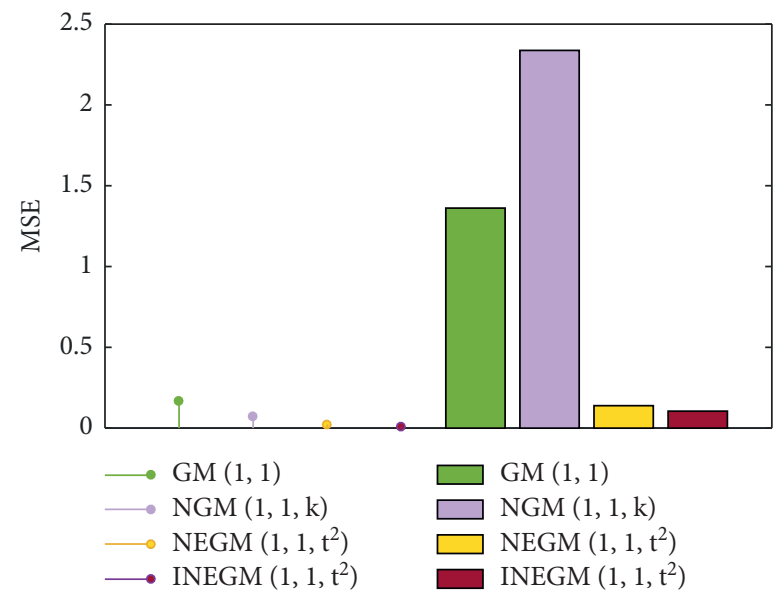

(d)

FIgUre 1: Continued. 


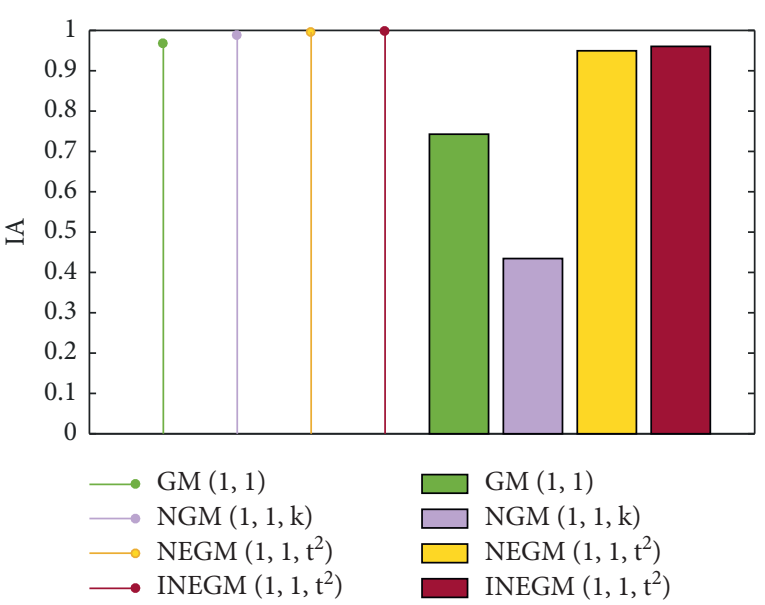

(e)

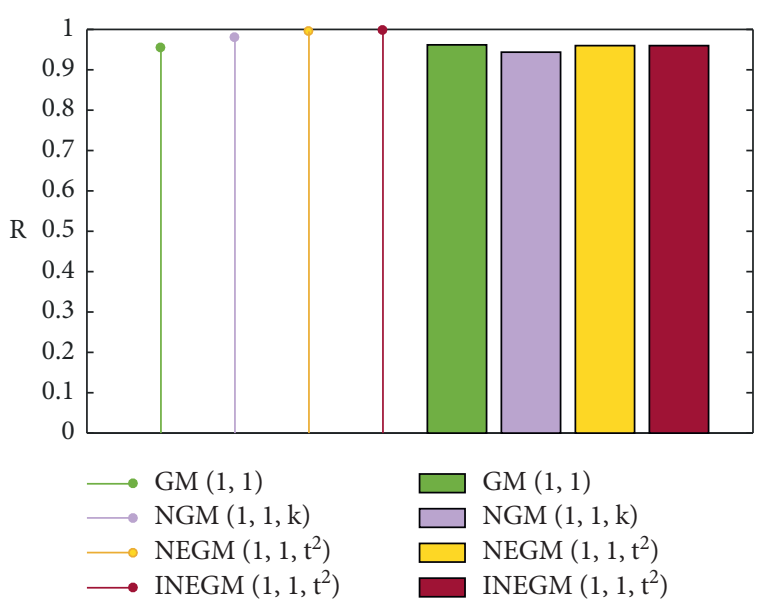

(f)

Figure 1: Errors by the competitive models in Case 1: (a) MAPE, (b) RMSPE, (c) MAE, (d) MSE, (e) IA, and (f) R.

TABLE 5: Building's settlement data in Case 2.

\begin{tabular}{lr}
\hline Time & Data \\
\hline 1 & 9.28 \\
25 & 10.71 \\
53 & 11.31 \\
83 & 11.64 \\
116 & 12.00 \\
147 & 12.23 \\
177 & 13.05 \\
237 & 13.16 \\
269 & 13.61 \\
355 & 13.94 \\
\hline
\end{tabular}

TABLE 6: Simulative and predictive values by the competitive models in Case 2.

\begin{tabular}{|c|c|c|c|c|c|c|c|c|c|}
\hline \multirow{2}{*}{ Time } & \multirow{2}{*}{ Data } & \multicolumn{2}{|c|}{ GM $(1,1)$} & \multicolumn{2}{|c|}{$\operatorname{NGM}(1,1, k)$} & \multicolumn{2}{|c|}{$\operatorname{NEGM}\left(1,1, t^{2}\right)$} & \multicolumn{2}{|c|}{$\operatorname{INEGM}\left(1,1, t^{2}\right)$} \\
\hline & & Value & APE (\%) & Value & APE (\%) & Value & APE (\%) & Value & APE (\%) \\
\hline \multicolumn{10}{|c|}{ In-sample } \\
\hline 1 & 9.28 & 9.28 & 0.00 & 9.28 & 0.00 & 9.28 & 0.00 & 9.28 & 0.00 \\
\hline 25 & 10.71 & 10.88 & 1.59 & 10.75 & 0.37 & 10.75 & 0.37 & 10.72 & 0.56 \\
\hline 53 & 11.31 & 11.19 & 1.06 & 11.27 & 0.35 & 1.30 & 0.09 & 11.30 & 0.11 \\
\hline 83 & 11.64 & 11.54 & 0.86 & 11.68 & 0.34 & 11.67 & 0.26 & 11.66 & 0.16 \\
\hline 116 & 12.00 & 11.93 & 0.58 & 12.00 & 0.00 & 11.97 & 0.25 & 12.28 & 0.39 \\
\hline 147 & 12.23 & 12.34 & 0.90 & 12.22 & 0.08 & 12.24 & 0.08 & 12.28 & 0.39 \\
\hline \multicolumn{10}{|c|}{ Out-of-sample } \\
\hline 177 & 13.05 & 12.75 & 2.30 & 12.36 & 5.29 & 12.48 & 5.29 & 12.56 & 3.72 \\
\hline 237 & 13.16 & 13.38 & 1.67 & 12.50 & 5.02 & 12.83 & 5.02 & 12.99 & 1.31 \\
\hline 269 & 13.61 & 14.04 & 3.16 & 12.58 & 7.57 & 13.18 & 7.57 & 13.42 & 1.40 \\
\hline 355 & 13.94 & 14.96 & 7.32 & 12.63 & 9.40 & 13.63 & 9.40 & 13.97 & 0.24 \\
\hline
\end{tabular}


TABle 7: Errors by the competitive models in Case 2.

\begin{tabular}{|c|c|c|c|c|}
\hline Index & GM $(1,1)$ & $\operatorname{NGM}(1,1, k)$ & $\operatorname{NEGM}\left(1,1, t^{2}\right)$ & $\operatorname{INEGM~}\left(1,1, t^{2}\right)$ \\
\hline \multicolumn{5}{|l|}{ In-sample } \\
\hline$M A P E(\%)$ & 0.9980 & 0.2305 & 0.2103 & 0.1861 \\
\hline RMSPE (\%) & 0.0111 & 0.0008 & 0.0006 & 0.0005 \\
\hline$M A E$ & 0.1140 & 0.0260 & 0.0240 & 0.0221 \\
\hline$M S E$ & 0.0141 & 0.0010 & 0.0007 & 0.0007 \\
\hline$I A$ & 0.9916 & 0.9994 & 0.9996 & 0.9996 \\
\hline$R$ & 0.9940 & 0.9996 & 0.9997 & 0.9997 \\
\hline \multicolumn{5}{|l|}{ Out-of-sample } \\
\hline MAPE (\%) & 3.6118 & 6.8170 & 3.0647 & 1.6697 \\
\hline RMSPE (\%) & 0.1790 & 0.4967 & 0.1007 & 0.2208 \\
\hline$M A E$ & 0.4925 & 0.9225 & 0.4100 & 0.0758 \\
\hline$M S E$ & 0.3409 & 0.9222 & 0.7818 & 0.0758 \\
\hline$I A$ & 0.7710 & 0.4260 & 0.7818 & 0.9065 \\
\hline$R$ & 0.9823 & 0.9142 & 0.9807 & 0.9815 \\
\hline
\end{tabular}

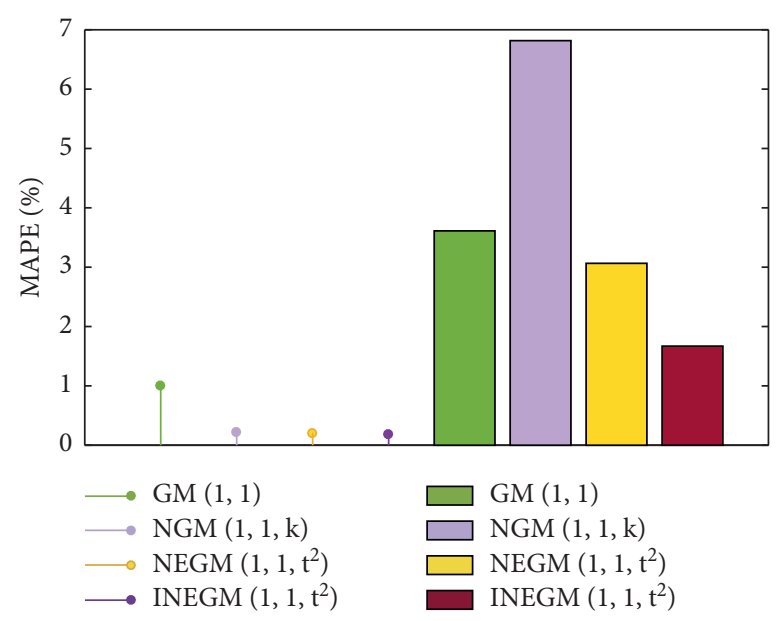

(a)

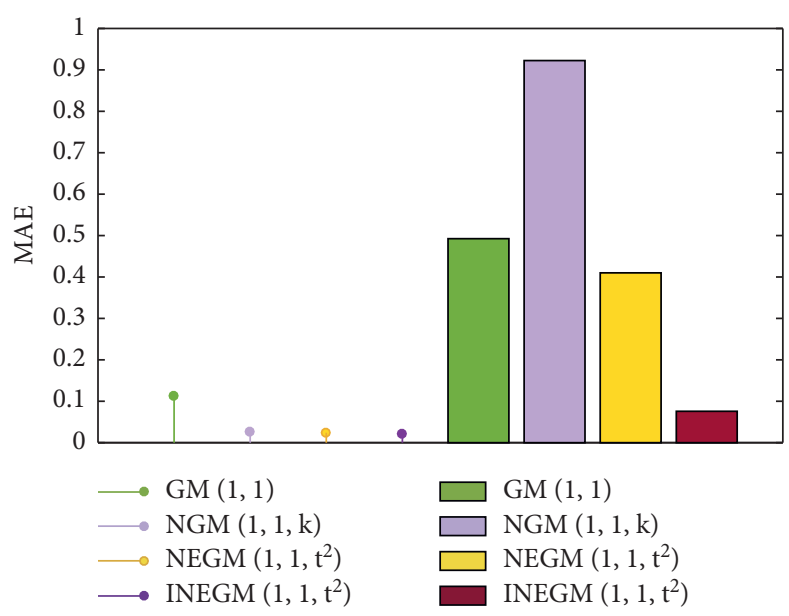

(c)

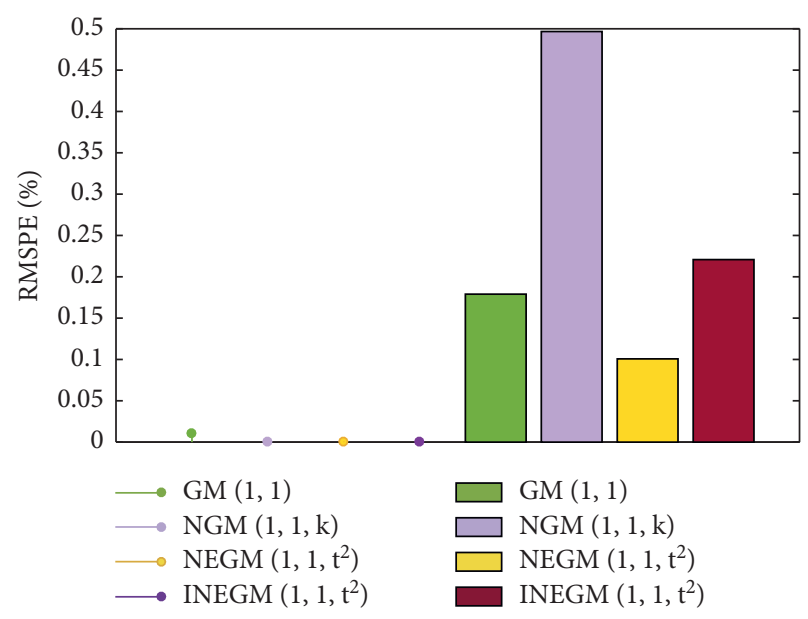

(b)

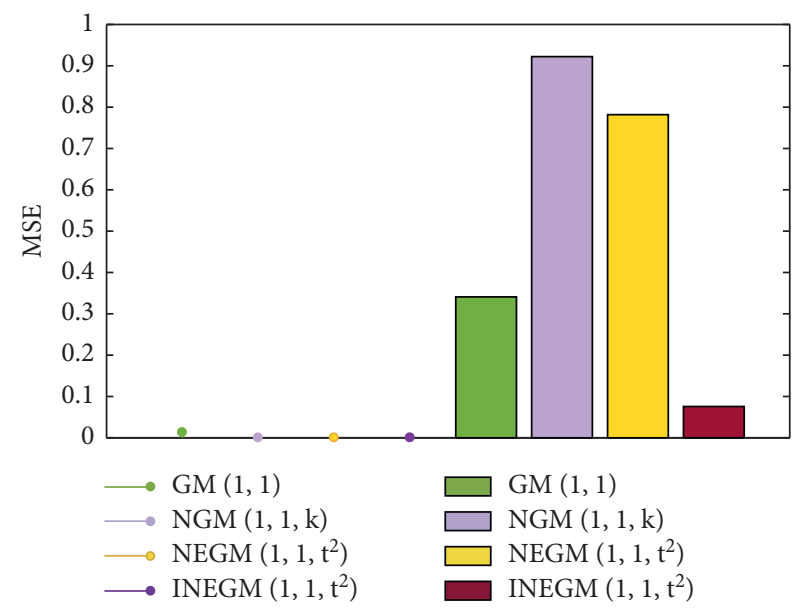

(d)

FIgURE 2: Continued. 


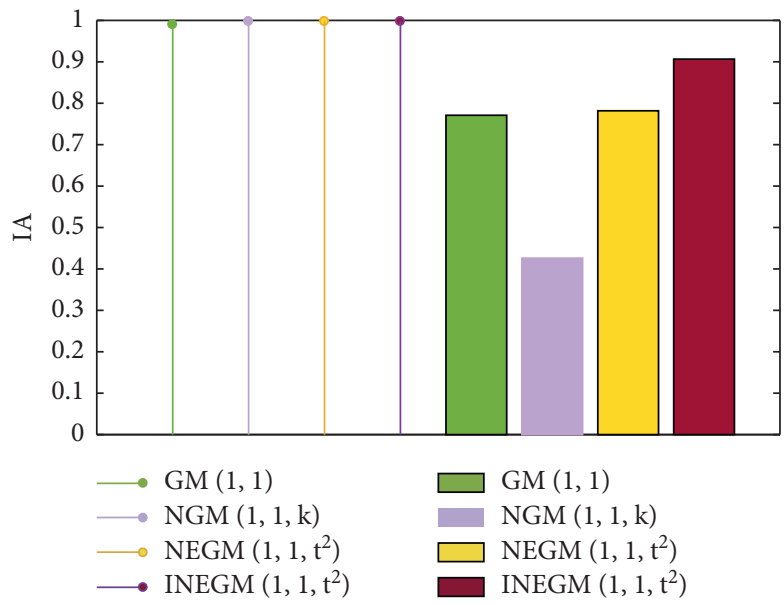

(e)

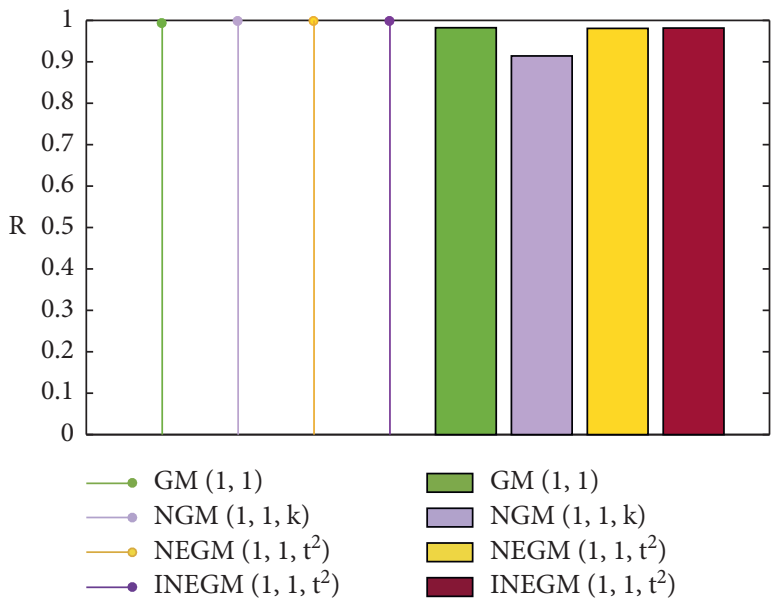

(f)

FIgure 2: Errors by the competitive models in Case 2: (a) MAPE, (b) RMSPE, (c) MAE, (d) MSE, (e) IA, and (f) R.

are better than the others. This fact indicates that the proposed model has a better prediction performance in this experiment.

\section{Conclusion}

Establishing a proper model for nonequidistant time-series forecasting has always been an issue that puzzles many scholars. In order to further broaden the development of nonequidistant grey prediction models, this paper develops a nonequidistant GM $\left(1,1, t^{2}\right)$ model (abbreviated as NEGM $\left.\left(1,1, t^{2}\right)\right)$ based on the previous literature.

To further improve the prediction accuracy of the $\operatorname{NEGM}\left(1,1, t^{2}\right)$ model, the modeling mechanism of the $\operatorname{NEGM}\left(1,1, t^{2}\right)$ model is deeply analyzed; it is known that, in the $\operatorname{NEGM}\left(1,1, t^{2}\right)$ model, the trapezoidal formula is used to calculate the area of curved trapezoid, thus producing an unacceptable error in such a transition process. To this end, we use the Simpson formula to optimize the $\operatorname{NEGM}\left(1,1, t^{2}\right)$ model based on the idea of function approximation and establish an improved NEGM $\left(1,1, t^{2}\right)$ model based on Simpson formula (abbreviated as INEGM $\left(1,1, t^{2}\right)$ ). To verify the feasibility and validity of the INEGM $\left(1,1, t^{2}\right)$ model, the INEGM $\left(1,1, t^{2}\right)$ model and three other grey prediction models are applied to two published cases. The results show that the accuracy of the INEGM $\left(1,1, t^{2}\right)$ model is higher than those of the NEGM $\left(1,1, t^{2}\right)$ model and two other prediction models. Therefore, the feasibility and validity of the INEGM $\left(1,1, t^{2}\right)$ model proposed in this paper are verified.

Although the advantages of the proposed model have been discussed, there are some limitations of the proposed model which should be considered in the following work; for example, this model is among the building blocks of the univariate model, potentially neglecting the relevant factors in practical applications. Meanwhile, combining the existing nonequidistant grey model with intelligent techniques merits further research.

\section{Data Availability}

The data used to support the findings of this study are included within the article.

\section{Conflicts of Interest}

The authors declare that they have no conflicts of interest.

\section{Acknowledgments}

This work was supported by Zhejiang College of Shanghai University of Finance and Economics for scientific research projects at the provincial and above levels and by the Academic Program of the Association for Science and Technology of Jinhua (201920).

\section{References}

[1] W. Zhou, X. Wu, S. Ding, and J. Pan, "Application of a novel discrete grey model for forecasting natural gas consumption: a case study of Jiangsu Province in China," Energy, vol. 200, Article ID 117443, 2020.

[2] W. Xie, W. Z. Wu, C. Liu, and J. Zhao, "Forecasting annual electricity consumption in China by employing a conformable fractional grey model in opposite direction," Energy, vol. 202, Article ID 117682, 2020.

[3] F. Yao, H. G. Müller, and J. L. Wang, "Functional linear regression analysis for longitudinal data," The Annals of Statistics, vol. 33, pp. 2873-2903, 2005.

[4] I. Škrjanc and D. Matko, "Fuzzy predictive functional control in the state space domain," Journal of Intelligent and Robotic Systems, vol. 31, no. 1, pp. 283-297, 2001.

[5] D. W. Hosmer Jr, S. Lemeshow, and R. X. Sturdivant, Applied Logistic Regression, Vol. 398, John Wiley \& Sons, Hoboken, NY, USA, 2013.

[6] D. R. Cox and V. Isham, "A simple spatial-temporal model of rainfall," A. Mathematical and Physical Sciences, vol. 415, no. 1849 , pp. 317-328, 1988. 
[7] K. R. Gabriel and J. Neumann, "A Markov chain model for daily rainfall occurrence at Tel Aviv," Quarterly Journal of the Royal Meteorological Society, vol. 88, no. 375, pp. 90-95, 1962.

[8] S. Gao, M. Zhou, Y. Wang, J. Cheng, H. Yachi, and J. Wang, "Dendritic neuron model with effective learning algorithms for classification approximation and prediction," IEEE Transactions on Neural Networks and Learning Systems, vol. 30, no. 2, pp. 601-614, 2018.

[9] T. Zhou, S. Gao, J. Wang, C. Chu, Y. Todo, and Z. Tang, "Financial time series prediction using a dendritic neuron model," Knowledge-Based Systems, vol. 105, pp. 214-224, 2016.

[10] J. A. K. Suykens and J. Vandewalle, "Least squares support vector machine classifiers," Neural Processing Letters, vol. 9, no. 3, pp. 293-300, 1999.

[11] J. Biamonte, P. Wittek, N. Pancotti, P. Rebentrost, N. Wiebe, and S. Lloyd, "Quantum machine learning," Nature, vol. 549, no. 7671, pp. 195-202, 2017.

[12] C. Zheng, W. Z. Wu, W. Xie, Q. Li, and T. Zhang, "Forecasting the hydroelectricity consumption of China by using a novel unbiased nonlinear grey Bernoulli model," Journal of Cleaner Production, vol. 278, Article ID 123903, 2021.

[13] W. Xie, W.-Z. Wu, T. Zhang, and Q. Li, "An optimized conformable fractional non-homogeneous gray model and its application," Communications in Statistics-Simulation and Computation, pp. 1-16, 2020.

[14] Z.-X. Wang and D.-J. Ye, "Forecasting Chinese carbon emissions from fossil energy consumption using non-linear grey multivariable models," Journal of Cleaner Production, vol. 142, pp. 600-612, 2017.

[15] B.-l. Wei, N.-m. Xie, and Y.-j. Yang, "Data-based structure selection for unified discrete grey prediction model," Expert Systems with Applications, vol. 136, pp. 264-275, 2019.

[16] X. Ma, X. Mei, W. Wu, X. Wu, and B. Zeng, "A novel fractional time delayed grey model with Grey Wolf Optimizer and its applications in forecasting the natural gas and coal consumption in Chongqing China," Energy, vol. 178, pp. 487-507, 2019.

[17] W. Wu, X. Ma, Y. Zhang, W. Li, and Y. Wang, "A novel conformable fractional non-homogeneous grey model for forecasting carbon dioxide emissions of BRICS countries," Science of the Total Environment, vol. 707, Article ID 135447, 2020.

[18] M. Xie, L. Wu, B. Li, and Z. Li, "A novel hybrid multivariate nonlinear grey model for forecasting the traffic-related emissions," Applied Mathematical Modelling, vol. 77, pp. 1242-1254, 2020.

[19] U. Şahin, "Forecasting of Turkey's greenhouse gas emissions using linear and nonlinear rolling metabolic grey model based on optimization," Journal of Cleaner Production, vol. 239, Article ID 118079, 2019.

[20] L. Liu, Y. Chen, and L. Wu, "The damping accumulated grey model and its application," Communications in Nonlinear Science and Numerical Simulation, vol. 95, Article ID 105665, 2021.

[21] D. Ju-Long, “Control problems of grey systems," Systems \& Control Letters, vol. 1, no. 5, pp. 288-294, 1982.

[22] W. Y. Qian, Y. G. Dang, and S. F. Liu, "Grey GM ( $111 t \alpha)$ model with time power and its application," Systems Engineering-Theory \& Practice, vol. 32, no. 10, pp. 2247-2252, 2012.

[23] L. Dang and W. Baolei, "Grey forecasting model with polynomial term and its optimization," Optimization, vol. 29, no. 3, pp. 58-69, 2017.
[24] B. Wei, N. Xie, and A. Hu, "Optimal solution for novel grey polynomial prediction model," Applied Mathematical Modelling, vol. 62, pp. 717-727, 2018.

[25] J. Cui, Y. G. Dang, and S. F. Liu, "Novel grey forecasting model and its modeling mechanism," Control and Decision, vol. 24, no. 11, pp. 1702-1706, 2009.

[26] F. B. Hildebrand, Introduction to Numerical Analysis, Courier Corporation, Chelmsford, MA, USA, 1987.

[27] Z. Yonglei and H. Xiufeng, "Improved discrete grey prediction model of ground settlement around excavation," Geotechnical Investigation \& Surveying, vol. 2, 2013.

[28] L. Xi, D. Song, N. Xu, and P. P. Xiong, "Research on optimization of non-equidistant GM (1 1 ) model based on the principle of new information priority," Control and Decision, vol. 34, pp. 2221-2228, 2019. 